\title{
Noncontact gravity compensator with magnetic fluid seals
}

\author{
Noriyuki HASEGAWA*, Hayato YOSHIOKA** and Hidenori SHINNO*** \\ *Department of Mechano-Micro Engineering, Tokyo Institute of Technology \\ 4259 Nagatsuta-cho, Midori-ku, Yokohama-shi, Kanagawa 226-8503, Japan \\ E-mail: Hasegawa.n.ac@m.titech.ac.jp \\ **Department of Mechanical and Control Engineering, Tokyo Institute of Technology \\ 2-12-1 Ookayama, Meguro-ku, Tokyo 152-8550, Japan \\ ***Precision and Intelligence Laboratory, Tokyo Institute of Technology \\ 4259 Nagatsuta-cho, Midori-ku, Yokohama-shi, Kanagawa 226-8503, Japan
}

\section{Received 29 February 2016}

\begin{abstract}
In vertical positioning systems, the friction force and the gravity load cause significant error. In order to minimize the effect of friction and gravity load, this paper presents the noncontact gravity compensator applying magnetic fluid seals. The structure of the magnetic fluid seals was proposed for the linear motion system, and the dimensions of the magnetic fluid seal components were determined based on the result of the magnetic field analysis using FEM. Magnetic field analysis was used in order to investigate the relationship between the seal capacity and varied dimensions. Based on the results of the analysis, several types of magnetic fluid seal units were manufactured, and the analytical results were compared with the results of the seal capacity evaluation experiment in order to confirm the validity of the magnetic field analysis. In addition, the gravity compensating system using the magnetic fluid seals was constructed and applied to a vertical positioning system. Positioning resolution was evaluated with stepwise positioning experiments. Tracking performance and the response of the pressure were evaluated by the continuous path control experiment. According to these experimental results, the effectiveness of the gravity compensator with magnetic fluid seal was confirmed.
\end{abstract}

Key words : Magnetic fluid seal, Gravity compensation, Linear motion system, Magnetic field analysis, Vertical positioning

\section{Introduction}

Industrial demands for generating complex shape have recently increased. However, most of the elaborated elements such as micro lens arrays or holographic optical elements are made of hard materials which cannot be fabricated by lithography (Kuriyagawa, 2009). Therefore, the machining systems which can machine such materials are required. In order to realize three-dimensional precision machining, such systems require not only horizontal positioning systems but also vertical positioning systems with nanometer positioning accuracy.

In a vertical positioning system, the gravity load of moving part affects the positioning accuracy negatively. This is because such systems require actuators to generate large force enough to support the gravity load while positioning. In order to exclude the effect of gravity, counterweights and counterbalances have been widely used so far (Beckert et al., 2001; Ohtsuka and Furukawa, 1990). Although these methods are effective for compensating the gravity load, they cause friction force. The friction force between the moving part and the surrounding fixed components may cause positioning error due to the nonlinear behavior. Friction force usually degrades the dynamic performance and positioning accuracy, and can also lead to steady state error (Mao et al., 2003). Therefore, the system which can compensate the gravity load in a perfect noncontact condition is required.

In order to provide a gravity compensation in a noncontact condition, the gravity compensating system using vacuum cylinder has been proposed (Takahashi et al., 2008). The system achieves the noncontact condition by forming narrow seal clearance. However, it requires high accuracy of dimension and ease of assemble to form a narrow seal 
clearance. Besides, it requires a vacuum pump to support large load because the outside air continuously flows through the seal clearance. Thus, it is necessary to apply the sealing element which achieves the ease of assemble with higher sealing performance on the gravity compensating system.

Accordingly, it is expected that magnetic fluid seals function well for solving these problems utilizing the characteristics of magnetic fluid. These seals keep differential pressure without solid friction with larger seal clearance compared to that of the narrow clearance seals mentioned above.

In this paper, the gravity compensator using magnetic fluid seals was proposed in order to realize the noncontact gravity compensation with much easier to assemble than applying narrow clearance seals, and to prevent from the inflow of the outside air. The structure of the magnetic fluid seals was reconsidered in order to apply the seals to a linear motion system referring to the results of magnetic field analysis. Finally, the experimental results confirmed that the effectiveness of the proposed system.

\section{Application of magnetic fluid seals for linear motion system}

Magnetic fluid is paramagnetic colloidal solution which consists of base liquid and paramagnetic particles covered with surface-active agent (Yamaguchi, 2011). Magnetic fluid moves and transforms affected by magnetic field.

Magnetic fluid seals are commonly applied to rotating systems. For example, transitions of vacuum chambers, bearings of hard disk drives. Magnetic fluid seals have advantages that there is low friction, low heating, no dust emission, and high airtightness (Taketomi and Chikazumi, 1988). Fig. 1 shows the general structure of a magnetic fluid seal for a rotating system. In order to retain the magnetic fluid in a seal clearance, the structure is designed to form magnetic circuit. Magnetic fluid seals for rotating shafts are composed of magnets, pole pieces, a rotating shaft and magnetic fluid. The pole pieces and the rotating shafts are made of magnetic materials. The magnets produce the magnetic flux, and the pole pieces concentrate the magnetic flux to the seal clearance. Then the magnetic circuits are led through these four components.

When the magnetic fluid seals which have the same structure as those of rotating system were applied in linear motion system, some problems occur such as leakage of the magnetic fluid at the seal clearance due to the movement, and positioning error due to the eddy current loss. To solve these problems, this study proposes the different structure from that of rotating systems. As shown in Fig. 2, the linear motion shaft is made of non-magnetic material to reduce the influence of magnetic force and the magnetic circuit does not pass through the shaft. Besides, the shaft has oil repellent surface in order to reduce the leakage of the magnetic fluid.

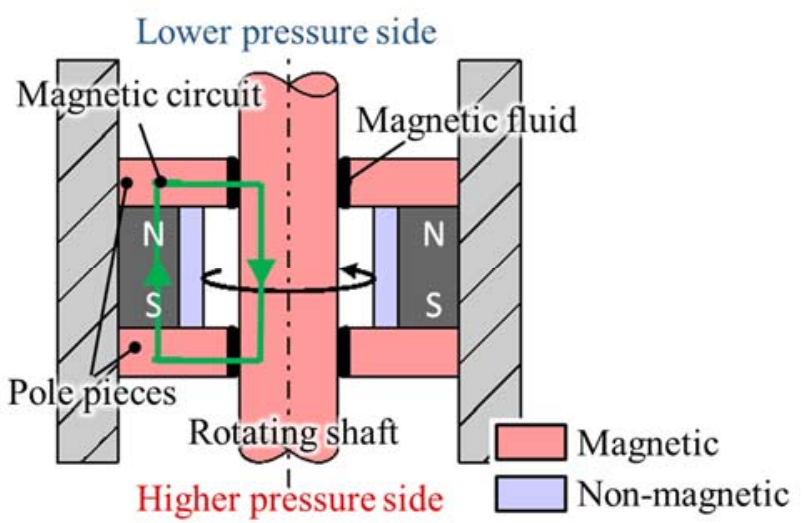

Fig. 1 Structure of a magnetic fluid seal for a rotating system.

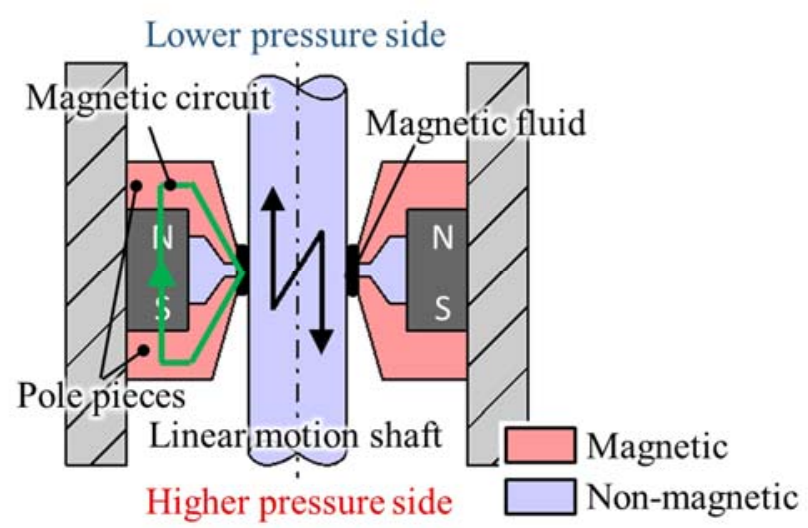

Fig. 2 Proposed structure of a magnetic fluid seal for a linear motion system.

\section{Design and performance evaluation of magnetic fluid seals}

The magnetic fluid seals for rotating systems are generally used under the condition that a multiple number of units in a row. Whereas, in a vertical positioning system, a large number of magnetic fluid seals cannot be embedded in a row because the increase of the number of the units leads to the increase of the length of the piston and the mass of the moving parts. Thus, each of the magnetic fluid seal units need to be designed to have high seal capacity. 
As the structure of the magnetic fluid seals for the linear motion systems differs from that of rotating systems, the shape of the pole pieces has to be designed as a different components from those of rotating systems.

\subsection{Estimation of the seal capacity using magnetic field analysis}

The seal capacity of single-stage static magnetic fluid seal can be generally represented as below (Rosensweig, 1985):

$$
\Delta p=\mu_{0} \int_{H_{2}}^{H_{3}} M \mathrm{~d} H
$$

where $\Delta p$ is the differential pressure between the both sides of the seal, $\mu_{0}$ is magnetic permeability of absolute vacuum, $H$ is the magnetic field intensity, $M$ is the magnetization, $H_{2}$ and $H_{3}$ is the minimum and the maximum values of magnetic field intensity.

Based on Eq. (1), the following equation is used for multiple-stage seals (De Volder and Reynaerts, 2009):

$$
\Delta p=M_{s}\left(B_{\max }-B_{\min }\right)
$$

where $M_{s}$ is the saturation magnetization of the magnetic fluid, $B_{\max }$ and $B_{\min }$ is the maximum and the minimum values of the magnetic flux density.

Based on Eq. (2), the seal capacity can be estimated by the distribution of the magnetic flux density. In the proposed structure, $\left(B_{\max }-B_{\min }\right)$ is the smallest on the surface of the piston. Therefore, the seal capacity depends on the magnetic flux density distribution on the surface of the piston as shown in Fig. 3.

In order to analyze the distribution of the magnetic flux density, the magnetic field analysis was conducted. The analysis model is shown in Fig. 3. $x$ is the width of the seal clearance, $d$ is the distance between two pole pieces, and $\alpha$ is the angle of the tapered side of the pole pieces. In the analysis, these three parameters were varied respectively, and then the results of the analysis were compared. During the analysis, the material of pole pieces was assumed to be SS400 steel. In order to analyze accurately, the alternation of magnetic permeability of the magnetic fluid has to be concerned (Park el al., 1994). However, it requires complex analysis because the magnetic intensity of the atmosphere and the magnetic permeability of the magnetic fluid depend on each other. In this study, the magnetic permeability of the magnetic fluid approximated to be 1.0 according to the report that the magnetic permeability of magnetic fluid tends towards 1.0 rapidly with the increase of the magnetic intensity (Zou, 1992).

One instance of the magnetic flux lines distributions which was obtained from the magnetic field analysis is shown in Fig. 4. It is observed that the magnetic flux is concentrated at the gap of two pole pieces. This means that the arrangement of these two pole pieces functions as desired.

The relationship between $\left(B_{\max }-B_{\min }\right)$ and each parameter is shown in Fig. 5. As indicated in Fig. 5, the maximum value of the magnetic flux density varies along the change of the width of the seal clearance $x$. As the magnetic permeability of the magnetic fluid is much less than that of pole pieces, it is natural that the magnetic flux density on the surface of the piston decreases when the seal clearance becomes wider. Thus, the relationship between the value $\left(B_{\max }-B_{\min }\right)$ and $x$ was obtained as shown in Fig. 6 (a).

According to Fig. 5, the maximum value of the magnetic flux density tends to decrease along the increase of the tapered angle $\alpha$. This is because the magnetic flux tends to have difficulty to pass through the tip of the pole pieces as the tapered angle $\alpha$ increases. On the other hand, the minimum value of the magnetic flux density tends to decrease along the increase of $\alpha$. These two tendency affect the variation of value $\left(B_{\max }-B_{\min }\right)$ oppositely, but the latter factor appeared stronger, so that the relationship between $\left(B_{\max }-B_{\min }\right)$ and $\alpha$ resulted as shown in Fig.6 (b).

The graphical form of the magnetic flux density distribution differs when the distance between two pole pieces is varied according to Fig. 5. This is because the magnetic flux lines which pass nearby the surface of the piston tend to decrease when the two pole pieces are too close or distant. Therefore, $\left(B_{\max }-B_{\min }\right)$ varies as shown in Fig. 6 (c).

Eventually, each parameters were determined as $x=100 \mu \mathrm{m}, d=400 \mu \mathrm{m}$, and $\alpha=30^{\circ}$ in order to bare a large differential pressure based on the results of the magnetic field analysis. 


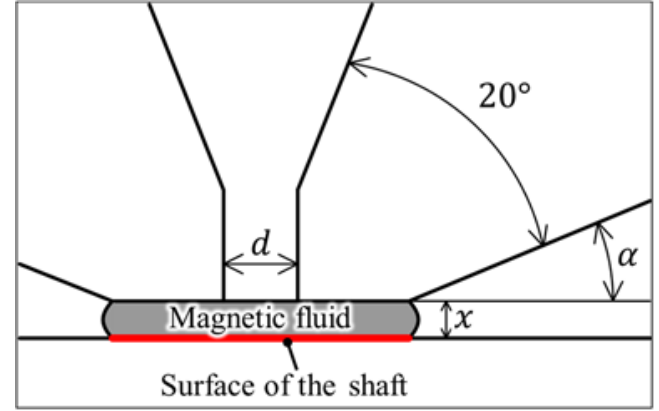

Fig. 3 Schematic diagram of each parameter of magnetic fluid seals.

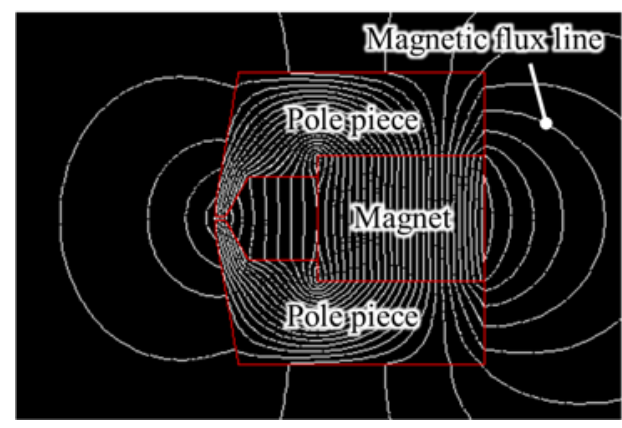

Fig. 4 Magnetic flux lines obtained from the magnetic field analysis.
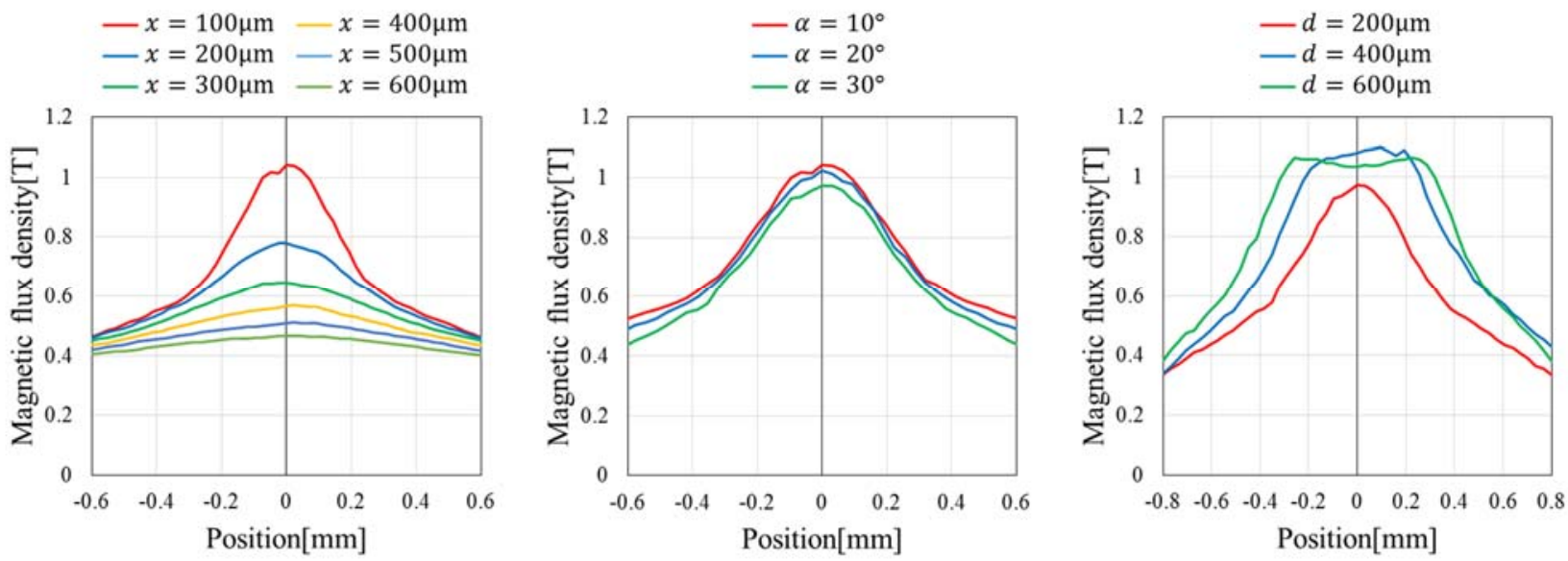

Fig. 5 Analytical results of the distribution of magnetic flux density on the surface of the piston for each varied parameters.

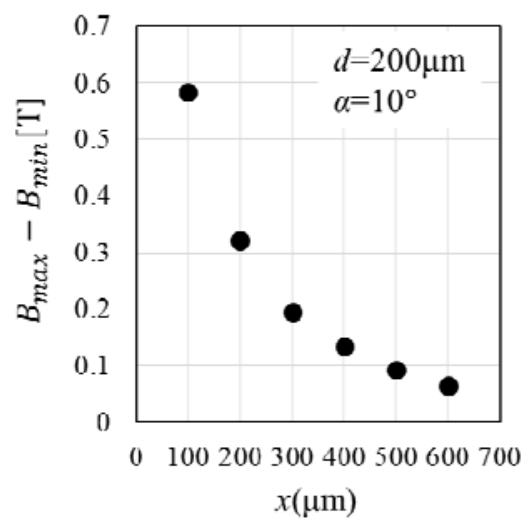

(a) Relationship between $\left(B_{\max }-B_{\min }\right)$ and $x$.

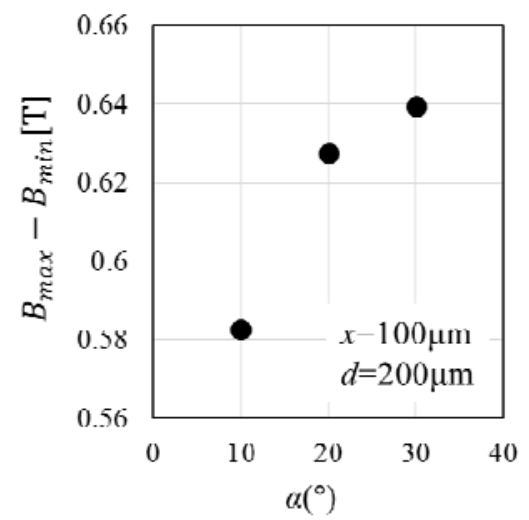

(b) Relationship between $\left(B_{\max }-B_{\min }\right)$ and $\alpha$.

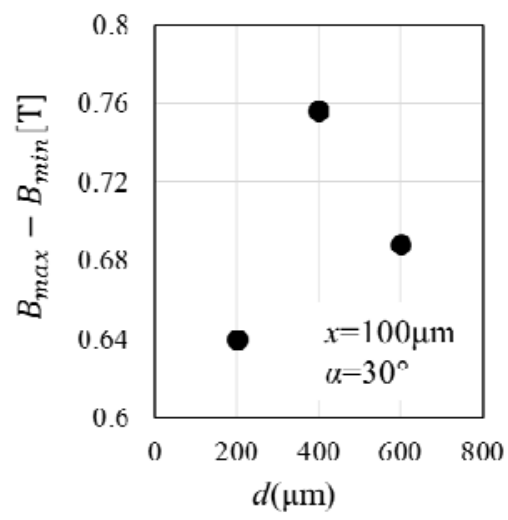

(c) Relationship between $\left(B_{\max }-B_{\min }\right)$ and $d$.

Fig. 6 Relationship between $\left(B_{\max }-B_{\min }\right)$ and each varied dimension. 


\subsection{Seal capacity evaluation}

In order to verify the analytical results, the evaluation experiments of the seal capacity were conducted. The appearance of the experimental device is shown in Fig. 7. Schematic diagram of the whole system is shown in Fig. 8. Three types of pole pieces were manufactured which differs in the tapered angle $\alpha$. Other dimensions mentioned above were fixed as $x=100 \mu \mathrm{m}, d=400 \mu \mathrm{m}$.

During the experiment, the inner pressure of the vacuum cylinder was gradually decreased by controlling the vacuum regulator, then the differential pressure gradually increased and finally a vacuum break occurred. In this study, the seal capacity can be defined as the maximum differential pressure before a vacuum break happens. The piston was kept at standstill during the measurement.

The experimental result is shown in Fig. 9 and the comparison of the seal capacity between the analytical results and the experimental results is shown in Fig. 10. According to Fig. 10, the analytical and the experimental results showed a qualitative agreement that the seal capacity increases along the increase of the tapered angle $\alpha$. However, the experimental values are larger than the analytical values. It can be thought that this difference were caused by other factor than magnetic flux density such as viscosity of the magnetic fluid. In this experiment, the effect of the viscosity might have appeared larger than that of rotating system because there is no flow inside the magnetic fluid in a steady state of linear motion system. In addition, since the actual distribution of magnetic fluid could not be observed, there is a possibility that the interface between the magnetic fluid and the piston was wider than assumed in Fig. 3.

Although the difference between the experimental results and the analytical results could be observed, the validity of the analysis was confirmed based on the qualitative agreement.

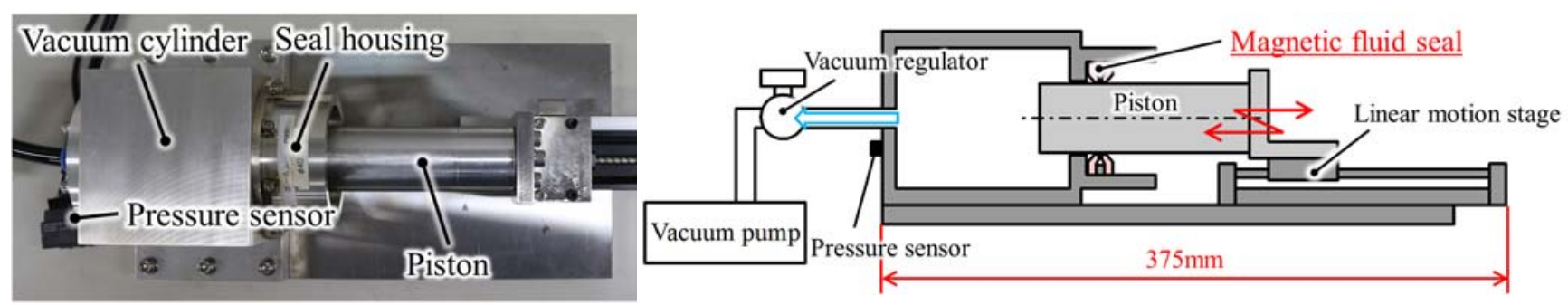

Fig. 7 Appearance of the experimental device for evaluating the seal capacity.

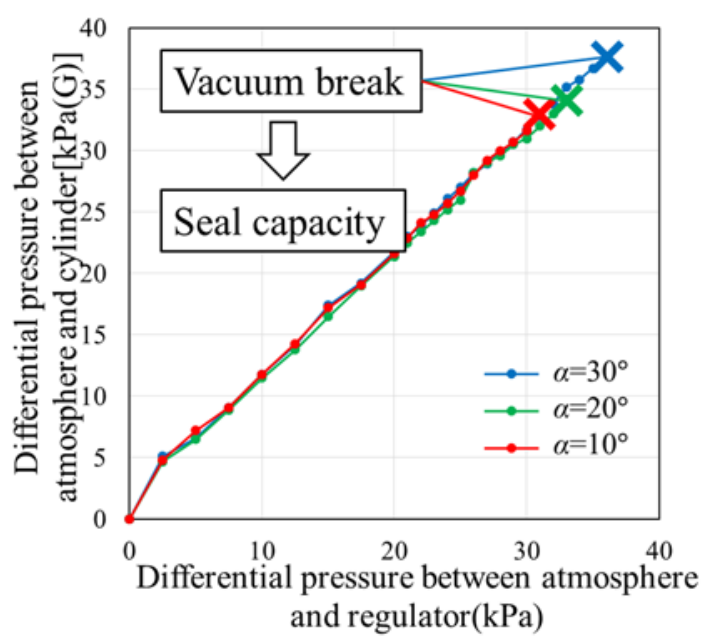

Fig. 9 Experimental results for finding out the seal capacity.
Fig. 8 Schematic diagram of the experimental device for the evaluation experiments of the seal capacity.

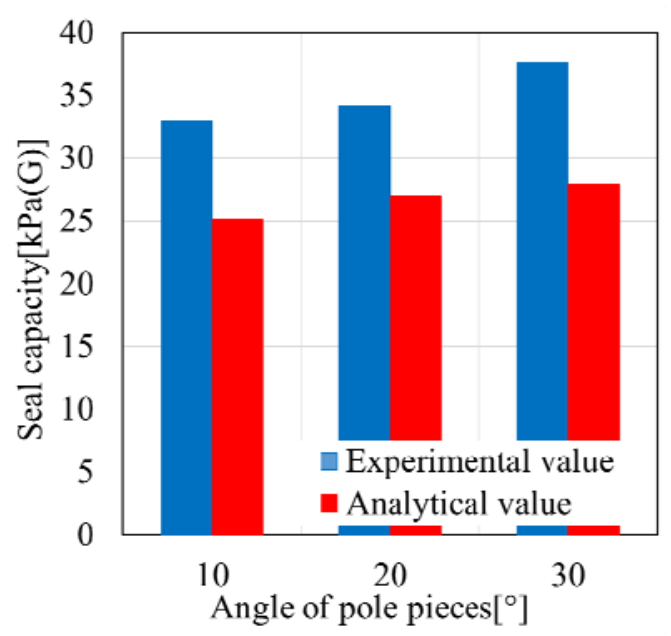

Fig. 10 Comparison between the experimental value and the analytical value of the seal capacity. 


\section{Performance evaluation of noncontact gravity compensating system 4.1 Experimental equipment}

In order to confirm the effectiveness of the gravity compensating system using the magnetic fluid seals, the vertical positioning system was constructed and then the gravity compensating system was applied to it. The developed vertical positioning system is shown in Fig. 11 and the block diagram of the system is shown in Fig. 12. In order to realize the vertical positioning system in a noncontact condition, both the voice coil motor and the aerostatic guide way were applied to the system. The actual position is measured by the optical linear encoder, and controlled by a PID controller using the encoder output as a feedback signal. The basic specification of the system is shown in Table 1.

During the positioning experiments mentioned below, the forward direction of positioning is defined as vertically upward.

Table 1 Basic specifications of the vertical positioning system.

\begin{tabular}{c|c|c|c|c}
\hline \hline Piston diameter & Mass of the moving part & Stroke length & Measurement resolution & Control frequency \\
\hline $40 \mathrm{~mm}$ & $5.9 \mathrm{~kg}$ & $50 \mathrm{~mm}$ & $10 \mathrm{~nm}$ & $10 \mathrm{kHz}$ \\
\hline
\end{tabular}
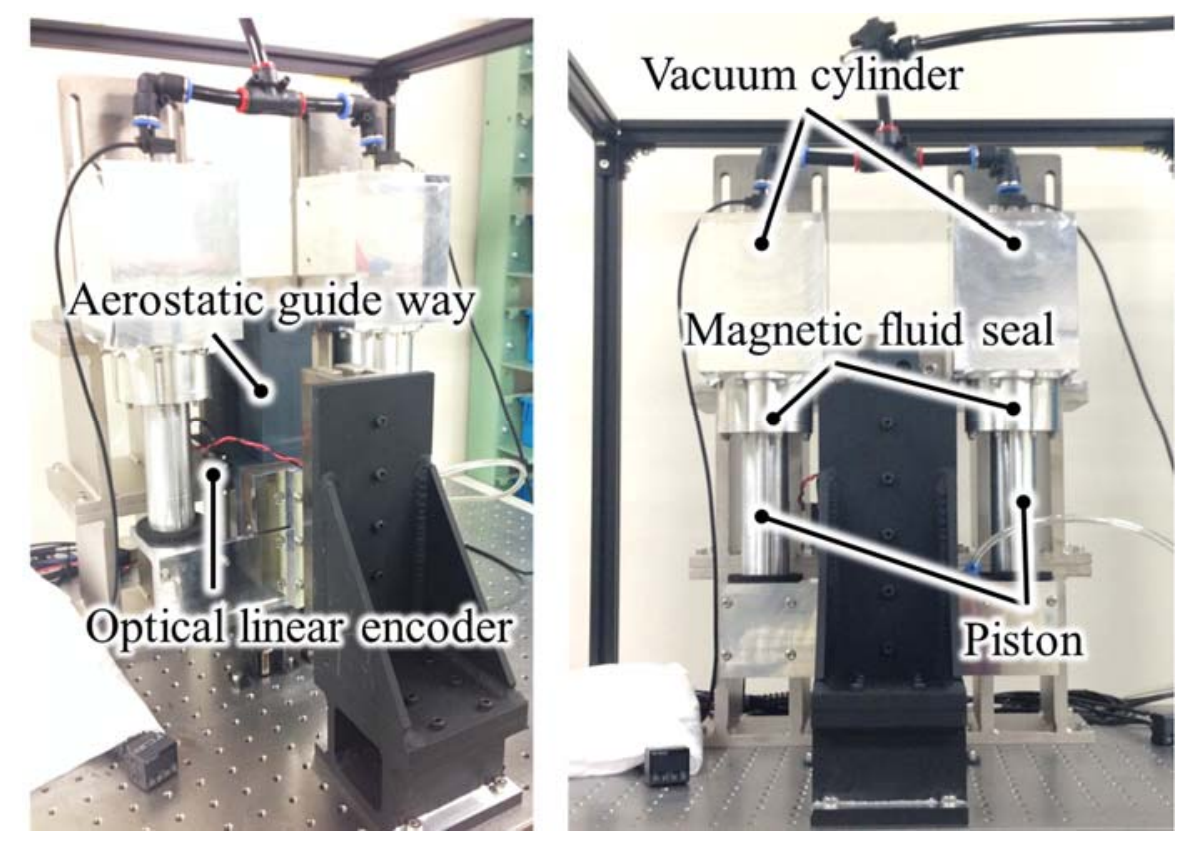

Fig. 11 Appearance of the constructed vertical positioning system.

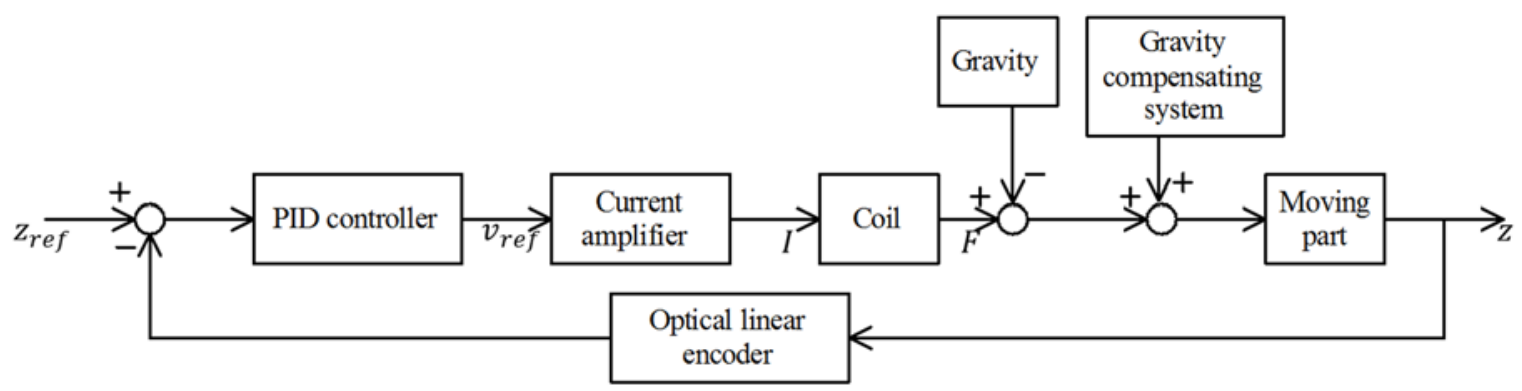

Fig. 12 Block diagram of the vertical positioning system. 


\subsection{Positioning resolution}

The stepwise positioning experiments were conducted in order to evaluate the positioning resolution of the system. The step height was varied, then each time response of the measured position was observed. Fig. 13 shows the actual system responses for each step height. According to the result, the step shape appeared clearly when the step height is larger than $50 \mathrm{~nm}$. Therefore, it can be said that this system has $50 \mathrm{~nm}$ positioning resolution. The function of gravity compensator contributed to the achievement by reducing the driving force of the voice coil motor. It is impossible to drive the moving part in this system if the gravity compensator is not applied because the estimated output current for supporting the gravity load is much larger than the rated current of the voice coil motor. In addition, the results show that the compensation force of the system is stable enough to achieve the step response without causing a significant vibration nor steady state error. The positioning resolution might appeared higher when a device which has higher measuring resolution was applied. In consequence, the results confirm that the proposed gravity compensating system can be applicable to a vertical positioning system and it is useful for the system using the voice coil motor.
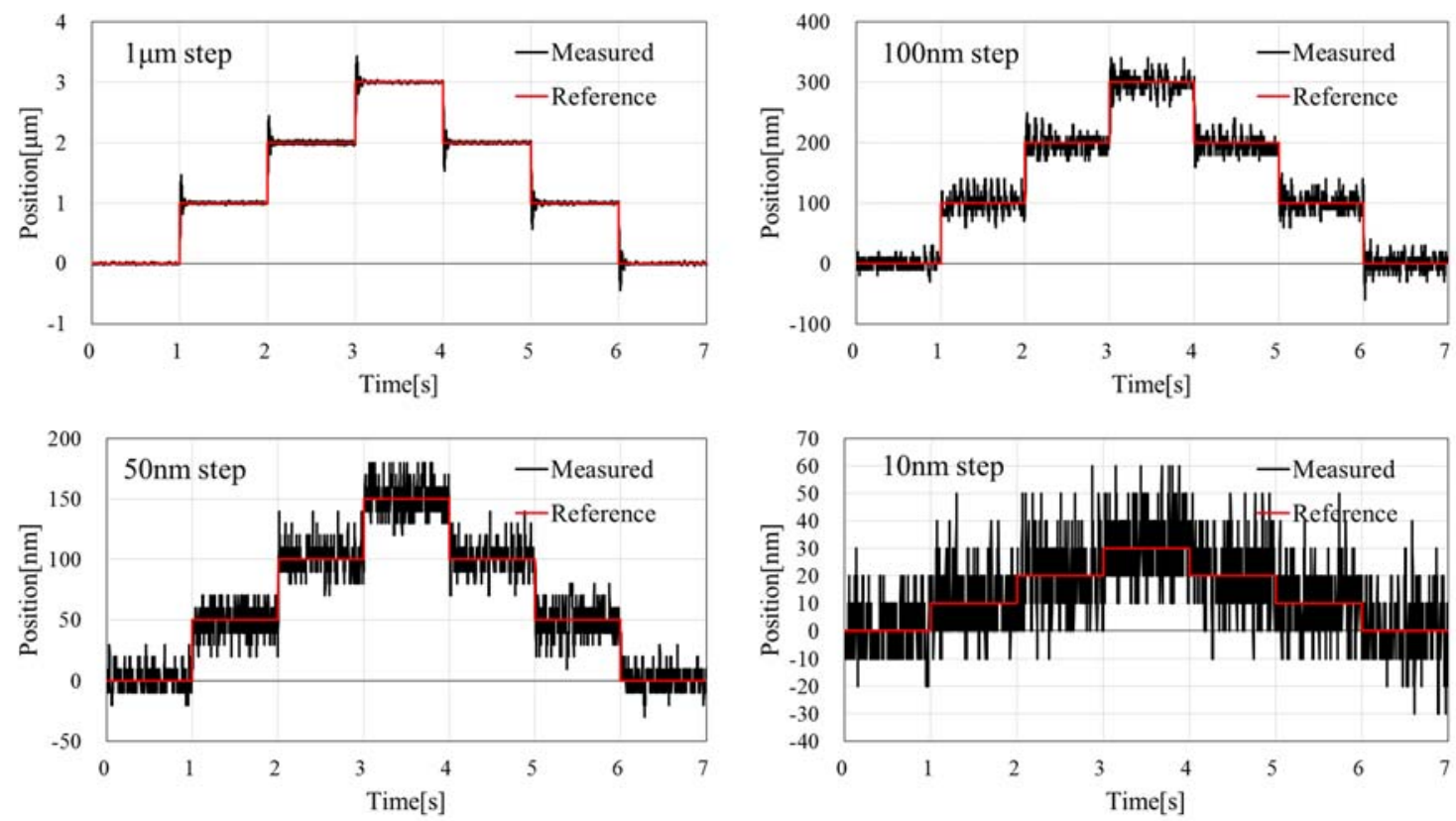

Fig. 13 Time response of the vertical positioning system for stepwise positioning.

\subsection{Response of continuous path control}

Figure 14 shows the time response of the position, the tracking error, and the inner pressure of the cylinder during the continuous path control. The tracking error was calculated by subtracting the reference value from the actual position. The behavior of the inner pressure shows that the gravity compensator worked normally without vacuum break during the $20 \mathrm{~mm}$ stroke movement. The tracking error can be kept less than $\pm 1.0 \mu \mathrm{m}$ range. It can be said that the magnetic fluid seals perform well during a long stroke positioning. According to the chart of the current output, the maximum value of the current output is $0.427 \mathrm{~A}$. 7.68A current output is estimated to be necessary in order to keep the $5.9 \mathrm{~kg}$ moving mass lifted without gravity compensation. Compared with this, it is confirmed that the proposed gravity compensating system enabled to control the moving part with much less current output than without gravity compensation. The reduction of the current output leads to the reduction of the exothermic from the coil. It is widely known that the heat generation causes positioning error because of thermal deformation of the system (Ito, 2010). In the proposed system, heat generation causes the serious deformation at several points such as the seal clearance and the clearance of the aerostatic guide way because of the difference of the thermal expansion coefficient between the materials of the structures. Those matters cause the undesirable friction between the moving part and surrounding structure. Furthermore, when applying this system to a machine tool, the heat generation also cause the change of the distance between the measuring point and the cutting point. In consequence, the heat generation makes it impossible to 
measure the actual position of the cutting point. Therefore, the proposed gravity compensator with magnetic fluid seals is effective to reduce the positioning error caused by the heat generation.

The primary possible causes of the tracking error are the output delay during accelerating or decelerating sections and the pressure fluctuation due to the movement of the pistons. It is thought to be effective to apply additional control to the system such as acceleration feedforward of pressure fluctuation feedforward.
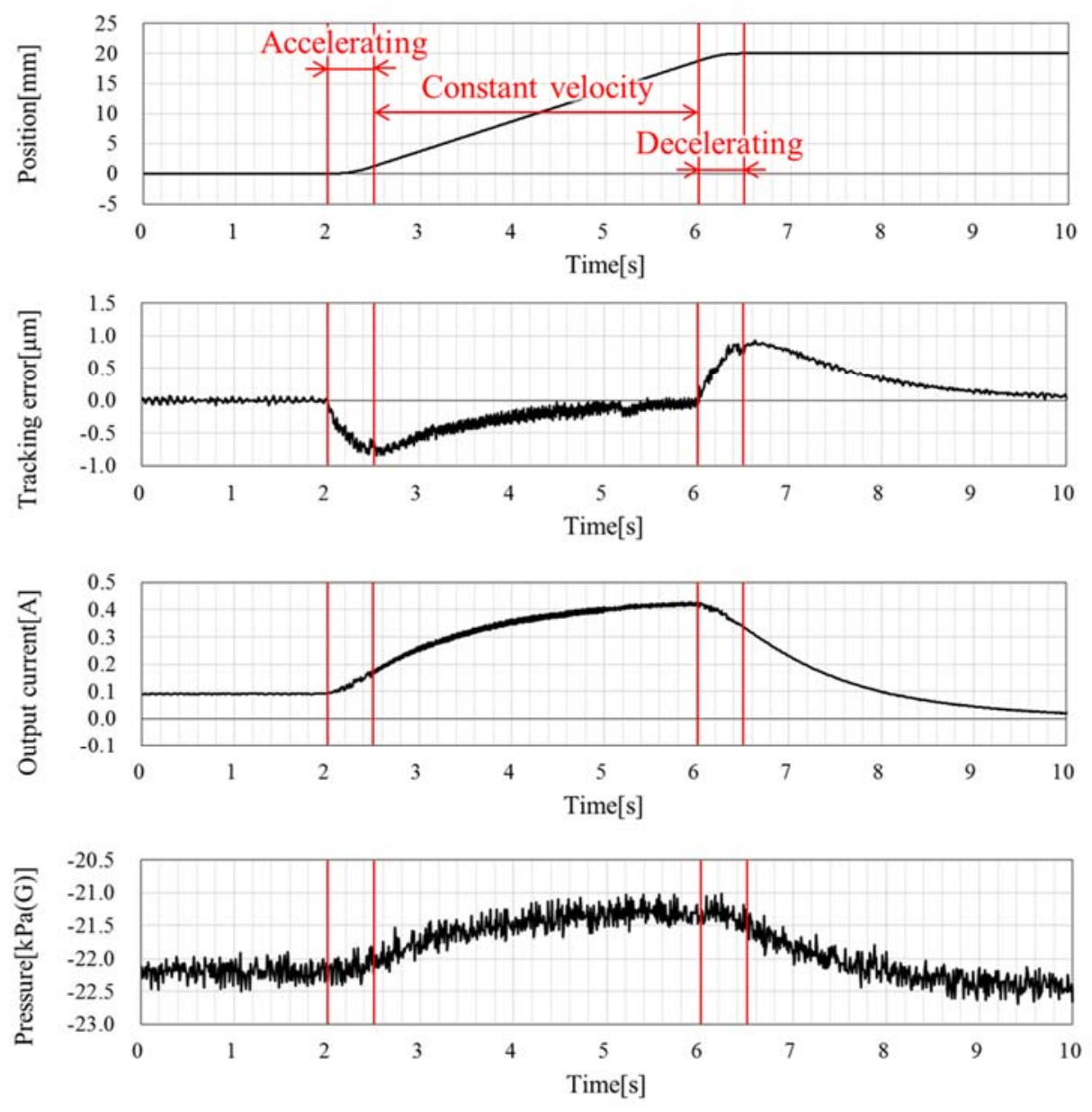

Fig. 14 The reference path and the time response of tracking error, output current, and the inner pressure of the cylinders during the continuous path control experiment.

\subsection{Behavior of the inner pressure of the cylinder due to the movement}

In order to improve the tracking performance of the system in the near future, the behavior of the inner pressure of the cylinder during the continuous path control was observed. The stroke direction and the velocity were varied, then the response of the pressure were compared.

Fig. 15 shows the reference path and the pressure responses for varied stroke velocity. According to the result, the magnitude of the pressure fluctuation tends to increase when the stroke velocity increases. This is because the pressure takes several seconds to settle back to the initial pressure set by the vacuum regulator.

Fig. 16 shows the comparison of the pressure responses for different stroke directions. The pressure behavior shows the similarity between upward and downward motion at the same stroke velocity. The behavior of the pressure depends on the flow rate of the air flow in the vacuum regulator or the other flows occur in some assembling clearance. In contrast to the similarity of the pressure behavior, the flow routes inside the regulator differ by upward and downward motion. Therefore, the similarity is not justified by the operation principle of the vacuum regulator.

The response of regulator has been widely studied in a previous research, and it is reported that the response predictions obtained from the dynamic model for a dome-loaded pressure regulator were experimentally validated. 
(Nabi et al., 2000). It is expected that the tracking error will be reduced when a pressure prediction is applied to a feedforward based on the dynamics of the vacuum regulator.
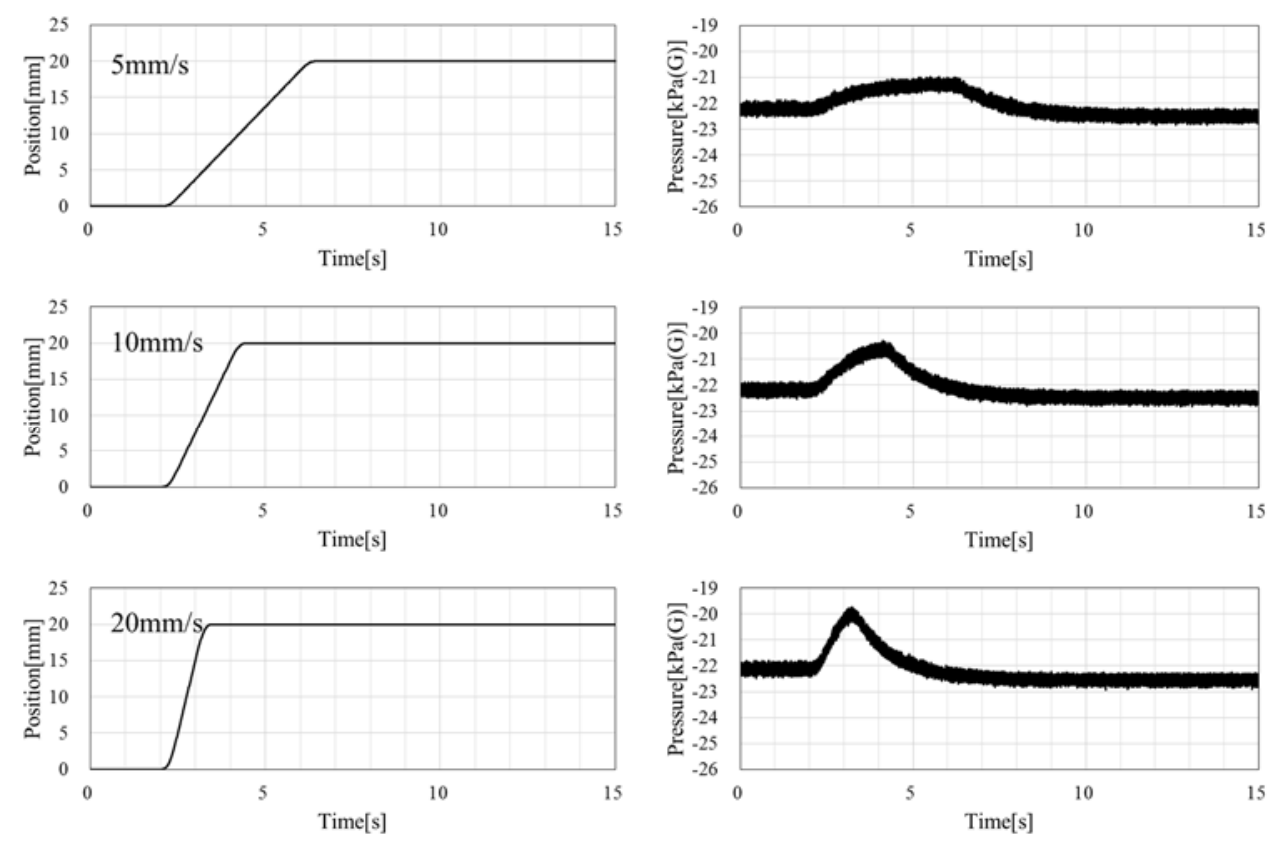

Fig. 15 Comparison of pressure responses for varied stroke velocity.
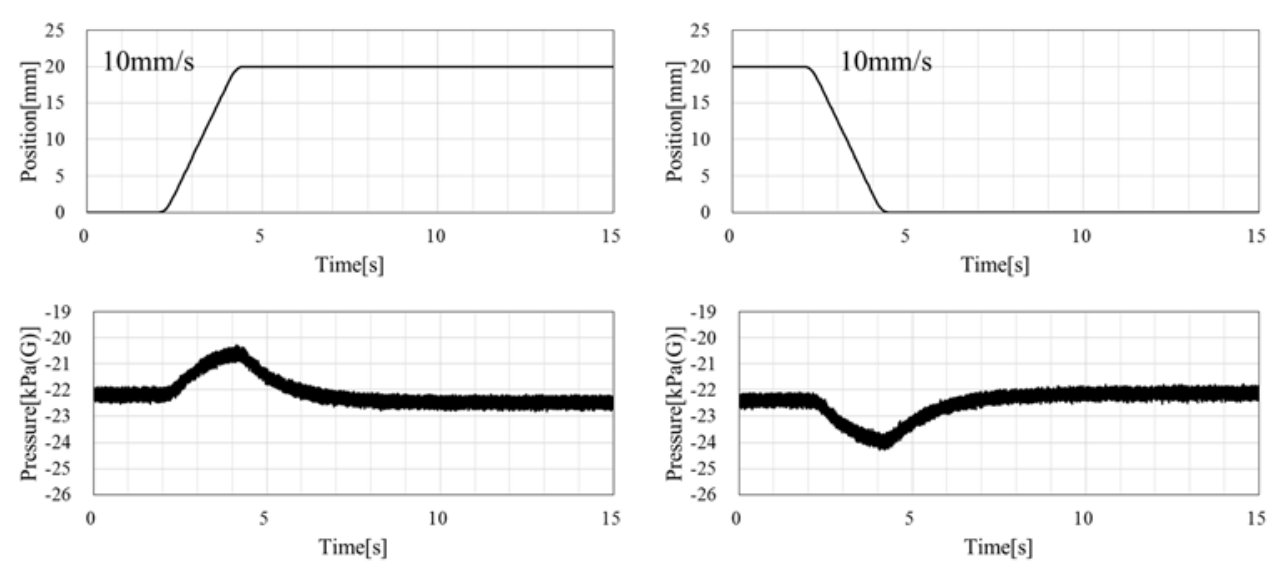

Fig. 16 Comparison of pressure responses for upward and downward $10 \mathrm{~mm} / \mathrm{s}$ strokes.

\section{Conclusions}

In this study, a gravity compensating system which works in noncontact condition was proposed. As a result, the following conclusions could be drawn:

1. The validity of the estimation of the seal capacity using the magnetic field analysis was confirmed by the comparison between the analytical value and the experimental value.

2. The noncontact gravity compensator with magnetic fluid seals could be applied to a vertical positioning system, and the $50 \mathrm{~nm}$ positioning resolution was obtained.

3. The gravity compensator provide the vertical stroke of $20 \mathrm{~mm}$ without a vacuum break. In addition, the gravity compensator achieved the positioning control with small current output. 


\section{Acknowledgement}

This research project was financially supported by the Japan Society for the Promotion of Science KAKENHI Grant-in-Aid for Scientific Research No.24226004.

\section{References}

Beckert, E., Hoffman, A. and Saffert, E., Development of a vertical wafer stage for high-vacuum applications (2001), Microelectronic Engineering, Vol.57-58, pp.207-212.

De Volder, M. and Reynaerts, D., Development of a hybrid ferrofluid seal technology for miniature pneumatic and hydraulic actuators (2009), Sensors and Actuators A, Vol.152, pp.234-240.

Ito, Y., Thermal deformation in machine tools (2010), p.32, McGraw-Hill.

Kuriyagawa, T., Future technologies of nano-precision micro/meso mechanical manufacturing (2009), Journal of the Japan Society for Precision Engineering, pp.62-63 (in Japanese).

Mao, J. Tachikawa, H. and Shimokohbe, A., Precision positioning of a DC-motor-driven aerostatic slide system (2003), Precision Engineering, Vol. 27, pp.32-41.

Ohtsuka, M. and Furukawa, M., The vertical traverse stage in vacuum condition (1990), JSME International Journal, Ser.3, Vol.33, No.1., pp.61-64.

Park, G., Kim, D., Hahn, S. and Lee, K., Numerical algorithm for analyzing the magnetic fluid seals (1994), IEEE Transactions on Magnetics, Vol.30, No.5, pp.3351-3354.

Rosensweig, R. E., Ferrohydrodynamics (1985), p.145, Cambridge University Press.

Takahashi, M., Yoshioka, H. and Shinno, H., A newly developed long-stroke vertical nano-motion platform with gravity compensator (2008), Journal of Advanced Mechanical Design, Systems, and Manufacturing, Vol.2, No.3, pp.356-365.

Taketomi, S. and Chikazumi, S., Magnetic Fluid (1988), p.17, Nikkan Kogyo Shimbunsha (In Japanese).

Yamaguchi, H., Magnetic Fluid (2011), pp.25-31, Morikita Publishing (in Japanese).

Zou, J. and Lu, Y., Numerical calculations for ferrofluid seals (1992), IEEE Transactions on Magnetics, Vol.28, No.6, pp.3367-3371. 